\title{
Enfermagem na prevenção mecânica de tromboembolismo venoso em pacientes cirúrgicos
}

\author{
Nursing in mechanical prevention of venous thromboembolism in surgical patients \\ La enfermería en la prevención mecánica del tromboembolismo venoso en pacientes quirúrgicos
}

Como citar este artigo:

Gomes ET, Assunção MCT, Lins EM, Püschel VAA. Nursing in mechanical prevention of venous thromboembolism in surgical patients. Rev Esc Enferm USP. 2021;55:e03738. https://doi.org/10.1590/S1980-220X2020002703738

\section{Eduardo Tavares Gomes ${ }^{1,2}$ \\ Marcone Cesar Tabosa Assunção ${ }^{1}$ \\ Esdras Marques Lins ${ }^{1}$ \\ Vilanice Alves de Araujo Püschel ${ }^{2}$}

${ }^{1}$ Universidade Federal de Pernambuco, Hospital das Clínicas, Recife, PE, Brasil.

${ }^{2}$ Universidade de São Paulo, Escola de

Enfermagem, Departamento de Enfermagem

Médico-cirúrgica, São Paulo, SP, Brasil.

\begin{abstract}
Objective: This theoretical study aims to discuss the role of nurses in the mechanical prevention of venous thromboembolism in surgical patients. Method: The study considered the updated versions of the main international guidelines and reviews on the topic. Non-pharmacological measures to prevent venous thromboembolism in surgical patients and the role of nurses are discussed. Results: It is important that surgical nurses include in their activities risk of assessments for venous thromboembolism and nonpharmacological prophylactic measures, based on scientific evidence and well-designed institutional protocols. Conclusion: Among the mechanical measures, the use of graduated compression stockings and intermittent pneumatic compression should be ensured by nurses.
\end{abstract}

\section{DESCRIPTORS}

Thromboembolism; Venous Thromboembolism; Patient Safety; Surgical Procedures, Operative; Operating Room Nursing.

\author{
Autor correspondente: \\ Eduardo Tavares Gomes \\ Escola de Enfermagem da \\ Universidade de São Paulo \\ Av. Dr. Enéas Carvalho de Aguiar, \\ 419, Cerqueira César \\ CEP 05403-000 - São Paulo, SP, Brasil \\ eduardotgomes@usp.br
}




\section{INTRODUÇÃO}

O Tromboembolismo Venoso (TEV), caracterizado principalmente pela trombose venosa profunda (TVP) e a embolia pulmonar (EP), representa séria complicação de saúde para pacientes clínicos e cirúrgicos internados, altos custos associados e maior tempo de internamento ${ }^{(1)}$. O TEV é a causa de morte evitável mais frequente nesses pacientes, além de ser a terceira causa mais costumeira de morte de origem cardiovascular, após doença coronariana e acidente cerebrovascular ${ }^{(1-3)}$.

Atualmente é reconhecido que os pacientes clínicos são de risco para TEV tanto quanto os que se submetem a procedimentos cirúrgicos. Contudo, alguns estudos indicam que os pacientes cirúrgicos recebem menos medidas de profilaxia adequadas que os clínicos ${ }^{(1-2)}$.

$\mathrm{Na}$ fisiopatologia do TEV estão presentes um ou mais dos seguintes fatores: hipercoagulabilidade, estase e injúria endotelial. A estase é gerada pela imobilização dos membros inferiores (MMII) ou pelo repouso por mais de 3 dias, comuns no período perioperatório, e aumenta o risco para o TEV. O mesmo é observado no trauma grave, no trauma raquimedular e na insuficiência cardíaca congestiva (ICC) $)^{(3-5)}$.

A frequência de complicações tromboembólicas, bem como suas consequências adversas e seu impacto econômico justificam a prioridade da tromboprofilaxia para a segurança do paciente cirúrgico, sendo fator significativo para reduzir a morbimortalidade a curto e longo prazo. Contudo, ainda existem dúvidas quanto a esse tipo de intervenção e quanto à forma ideal de entrega, embora haja transcorrido mais de cinco décadas em que se demonstrou que a profilaxia específica poderia reduzir o TEV, e quase três décadas após a elaboração da primeira diretriz baseada em evidências para nortear as práticas da profilaxia dessa doença ${ }^{(3-5)}$.

Apesar da existência de diretrizes para guiar a profilaxia do TEV, a adesão a essas diretrizes ainda não é a ideal, aumentando o número de casos de pacientes de alto risco que poderiam ter evitado esse evento se recebessem os cuidados adequados. Estima-se que a profilaxia mecânica seja ainda menos prescrita e utilizada adequadamente ${ }^{(6)}$.

O presente estudo teórico tem por objetivo discorrer sobre o papel do enfermeiro na prevenção mecânica do TEV em pacientes cirúrgicos, considerando os principais guidelines internacionais e revisões acerca do tema $a^{(2,4-5,7-8)}$.

Os guidelines que foram considerados são referenciais clássicos na área e que foram atualizados nos últimos $\operatorname{anos}^{(2,4-5,7-8)}$. Foram excluídos guidelines que apresentassem apenas diretrizes sobre a profilaxia farmacológica. O papel do enfermeiro é discutido em uma sessão, tendo por referência a taxonomia Nursing Interventions Classification (NIC) e um guideline da Associação Norte-Americana de Enfermeiros Perioperatórios (AORN), considerando que não há diretrizes nacionais ${ }^{(9)}$.

\section{Profillaxia meCÂNICA PARA O tromboembolismo VenOSO}

Os principais métodos não farmacológicos de profilaxia para o TEV incluem o uso de meias elásticas de compressão graduada (MECG), a compressão pneumática intermitente
(CPI) e bombas venosas para pés, a movimentação passiva e ativa dos MMII, além da deambulação precoce ${ }^{(3-4)}$. Embora esses métodos não elevem o risco de sangramento, são escassas as pesquisas sobre tais práticas, de acordo com uma ampla revisão de literatura ${ }^{(3)}$. Atualmente, as evidências indicam seu uso para pacientes com alto risco de sangramento ou combinadas com profilaxia farmacológica, para tentar aumentar sua efetividade ${ }^{(3)}$.

A diretriz do American College of Chest Physicians (ACCP) e o guideline europeu sobre profilaxia perioperatória para o TEV ainda consideram a profilaxia mecânica como um tema controverso ${ }^{(3,7)}$. O guideline reconhece haver poucos estudos sobre MECG e CPI, contudo, registra que há significância clínica reconhecida na prática, em relação à associação desses métodos com as estratégias farmacológicas, sendo a prática mais adotada nos serviços de cirurgia atualmente ${ }^{(5)}$.

Há poucos estudos sobre a efetividade do uso de MECG na maioria das especialidades cirúrgicas em relação a prevenção do TEV, mas há evidências que seu uso tenha resultados significativos em alguns cenários, como cirurgias plásticas, ortopédicas, cardíacas e neurológicas ${ }^{(3,10-11)}$. Uma revisão sistemática concluiu que não foi possível evidenciar o seu benefício na proteção para embolia pulmonar, mas ficou demonstrado o efeito preventivo sobre a TVP( ${ }^{(8)}$.

A MECG deve ter um gradiente de pressão, sendo mais elevada a pressão distal, próximo ao pé, e menor nas proximidades da coxa, geralmente com compressão mínima de $20 \mathrm{mmHg}{ }^{(12)}$. Meias acima do joelho ocasionam maior desconforto e são de difícil manuseio para realizar os cuidados diários. $\mathrm{Na}$ altura do joelho e da coxa costumam ser mais bem toleradas e eficazes na prevenção da TVP ${ }^{(12-14)}$. As MECG podem ser classificadas em suaves $(<15 \mathrm{mmHg})$, para descanso e prevenção de varizes; de compressão média (20 a 30 $\mathrm{mmHg}$ ) ou elásticas terapêuticas, para prevenção de TVP; de alta compressão ou antitrombo (30 a $40 \mathrm{mmHg}$ ), para síndrome pós-trombótica e insuficiência venosa crônica grave ${ }^{(15)}$.

As controvérsias no uso das MECG se relacionam ao seu uso isolado e à possibilidade de aumento de lesões de pele $\mathrm{e}^{(5)}$. É recomendado não fazer uso das MECG sem medidas farmacológicas, além de se observar protocolo seguro de avaliação permanente da pele, dos pulsos periféricos e da coloração das extremidades durante a sua utilização ${ }^{(5)}$. Além disso, calor gerado pelo uso das MECG pode fragilizar a pele e favorecer lesões e infecções. O enfermeiro deve ter essa rotina de avaliação enquanto o paciente estiver em uso de MECG e CPI, principalmente observando regiões de proeminências ósseas. Nos pacientes com diminuição da sensibilidade nos membros, deve ser verificada a presença de palidez na pele, perda da integridade, marcações pelo excesso de compressão, relato de dor ou desconforto ${ }^{(12,15-16)}$.

Assim como as meias elásticas, a CPI parece ser mais efetiva na prevenção de TVP em relação à EP, também no pós-operatório ${ }^{(3,17-19)}$. Quando comparadas com as meias, a CPI tem se mostrado com melhores resultados ${ }^{(7,11,20-21)}$. A CPI diminui a estase por comprimir repetidamente o membro, com intervalos periódicos, a uma pressão de inflação de $35-40 \mathrm{mmHg}$ e incremento na velocidade do fluxo de $180 \%$ a $240 \%{ }^{(15)}$. 
As diretrizes europeias contraindicam o uso de MECG isolada para pacientes de risco intermediário e alto e orientam que, para os pacientes que não podem receber profilaxia farmacológica, é mais fortemente indicado o uso da CPI que as meias elásticas ${ }^{(5)}$. Também são contraindicadas MECG e CPI de rotina em pacientes em uso de farmacoprofilaxia, exceto os que sejam considerados de alto risco para $\mathrm{TEV}^{(5)}$. A CPI combinada com agentes farmacológicos pode diminuir o risco de TEV, mas aumentar o risco de sangramento, quando comparada apenas com o uso isolado da CPI. E, ainda, pode diminuir o risco de EP em comparação à profilaxia farmacológica isolada ${ }^{(7,11,21)}$. São contraindicações ao uso da CPI: edema agudo de pulmão; TVP dos MMII; doença arterial periférica grave nos MMII; cirurgia de bypass da aorta e das artérias dos MMII; dermatite grave; úlceras cutâneas; enxerto recente de pele; neuropatia periférica e edema grave; ou presença de deformidade dos MMII ou cirurgias que impeçam o uso do recurso ${ }^{(12-13)}$.

\section{Protocolos InSTITUCIONAIS PARA O USO DE PROFILAXIA MECÂNICA PARA TROMBOEMBOLISMO VENOSO DO PACIENTE CIRÚRGICO}

O ACCP tem publicado diretrizes para tratamento e prevenção do TEV que vêm sendo utilizadas para elaboração de protocolos institucionais no mundo inteiro, tendo sido reforçadas pelas diretrizes europeias ${ }^{(5,11)}$. Nessa diretriz fica claro que os hospitais devem ter seus protocolos para profilaxia do TEV instituídos e difundidos entre o time cirúrgico, considerando a associação entre medidas farmacológicas ou não, destacando-se o uso de prevenção mecânica, principalmente para pacientes que têm anticoagulação contraindicada ${ }^{(5)}$.

Considerando as evidências e diretrizes, os protocolos institucionais devem iniciar pela recomendação da estratificação de risco de cada paciente para o TEV, que deverá nortear as ações e medidas não farmacológicas ${ }^{(3)}$. É significativo o número de pacientes de baixo risco para TEV que recebem intervenções desnecessárias, bem como o número de pacientes de alto e altíssimo risco que deixam de recebê-las ${ }^{(2)}$.

As instituições de saúde devem conhecer o perfil de pacientes em função dos estratos de riscos, com o intuito de adotar medidas para a correta profilaxia e, inclusive, alocar recursos de acordo com as melhores evidências( ${ }^{(6)}$. Embora o uso de MECG e CPI possa diminuir os custos considerando-se o valor do tratamento das TEV preveníveis, instaurar essas medidas ocasiona custo aos serviços de saúde. No sistema público, os custos estão relacionados ao custeio de meias para os pacientes de alto e altíssimo risco, bem como de dispositivos de compressão que têm compressores descartáveis e botas descartáveis.

O estudo mundial ENDORSE, realizado em 32 países, avaliou a prevalência de pacientes internados com risco para TEV e a proporção dos que receberam a correta profilaxia em 358 hospitais, concluindo que a profilaxia para TEV é mundialmente subutilizada, visto que apenas aproximadamente metade desses pacientes recebeu a profilaxia recomendada pelas diretrizes do $\mathrm{ACCP}^{(22-23)}$. Embora o estudo em questão tenha sido realizado há mais de uma década, estudos mais recentes vêm mostrando que o quadro não mudou como deveria ${ }^{(1,3,6)}$.

O modelo de Caprini é o mais amplamente difundido para a estratificação de risco ${ }^{(6)}$. Nesse modelo a avaliação é individualizada, sendo composta de fatores de risco que correspondem à pontuação de 1 a 5 pontos, além de uma pontuação específica para mulheres - de 1 ponto - quando elas apresentam uso de anticoncepcional ou terapia de reposição hormonal, gravidez ou pós-parto, prematuridade com toxemia ou desenvolvimento restrito ${ }^{(6,24)}$. De acordo com o escore alcançado pelo paciente na avaliação, esse é classificado em risco, sendo: Baixo, de 0 e 1 ponto; Moderado, 2 pontos; Alto, de 3 e 4 pontos; Altíssimo, com 5 ou mais pontos $^{(6,24)}$. A diretriz do ACCP recomenda profilaxia mecânica para pacientes de moderado e alto risco, quando houver contraindicação da profilaxia farmacológica ${ }^{(7)}$.

No Brasil vem sendo amplamente utilizada a classificação simplificada de avaliação de risco de TEV para pacientes cirúrgicos ${ }^{(2,25)}$, descrita a seguir.

- Baixo risco: Operações em pacientes de menos de 40 anos, sem outros fatores de risco; operações menores (de menos de 30 minutos e sem necessidade de repouso prolongado) em pacientes de mais de 40 anos, sem outro risco que não idade; trauma menor;

- Risco moderado: Cirurgia maior (geral, urológica ou ginecológica) em pacientes de 40 anos a 60 anos, sem fatores adicionais de risco, ou em pacientes de menos de 40 anos tomando estrógenos;

- Alto risco: Cirurgia geral em pacientes de mais de 60 anos ou em pacientes de 40 a 60 anos, com fatores de risco adicionais; cirurgia maior em pacientes com história de TVP ou EP pregressa ou trombofilia; grandes amputações; cirurgias ortopédicas maiores; cirurgias maiores em pacientes com neoplasias malignas; cirurgias maiores em pacientes com outros estados de hipercoagulabilidade; traumas múltiplos com fraturas de pélvis, quadril ou membros inferiores.

O Hospital Johns Hopkins, em Baltimore, nos Estados Unidos, iniciou em 2005 um time colaborativo para a prevenção do $\mathrm{TEV}^{(26)}$. A equipe multidisciplinar fez a revisão de protocolos e atualização permanente, elaborou estratégias de educação para os profissionais do hospital e a realização de ações de busca ativa, bem como auditoria para avaliar a melhora de processos e resultados. A prescrição adequada da tromboprofilaxia subiu de $26 \%$ para $80 \%$ dos $\operatorname{casos}^{(26)}$. A elaboração de uma ferramenta computadorizada de suporte para decisão e para inclusão de profissionais de várias áreas envolvidas garantiram o sucesso da estratégia no hospital ${ }^{(26)}$.

Um estudo de revisão apontou para o fato de que além dos alertas em prontuários eletrônicos, alertas humanos por farmacêuticos e enfermeiros, em estratégia de dupla ou tripla checagem, asseguram melhor prescrição ${ }^{(27)}$. O envolvimento de mais atores em treinamentos, desenvolvimento e implantação de protocolos melhora a qualidade da prescrição de profilaxia, devendo a enfermagem estar envolvida em todo processo ${ }^{(27-29)}$. 


\section{AÇÕES DE ENFERMAGEM NA PREVENÇÃO DO}

\section{TROMBOEMBOLISMO}

A avaliação de enfermagem deve começar no período pré-operatório e ser contínua até o pós-operatório. A Sistematização da Assistência de Enfermagem Perioperatória deve incluir a avaliação contínua do risco para TEV, bem como o registro dos Diagnósticos e Intervenções de Enfermagem correlatos. A orientação do paciente deve começar em consulta de enfermagem pré-operatória ambulatorial ou no internamento, incluindo a indicação do uso de meias, quando aplicável. $\mathrm{Na}$ admissão ao centro cirúrgico, o enfermeiro deve planejar a aplicação de CPI de acordo com a disponibilidade de compressores, o número de cirurgias e a indicação pelo risco. Por fim, no pós-operatório deve ser assegurada a manutenção desses recursos até a deambulação espontânea.

Em ampla revisão da literatura sobre a atuação da enfermagem na prevenção do TEV foram identificados estudos que evidenciaram ações concernentes: à avaliação e à estratificação do risco de TEV (28,5\%), à terapia compressiva (14,3\%), à eletroestimulação (14,3\%), ao posicionamento dos MMII (14,3\%), aos exercícios de amplitude de movimento $(14,3 \%)$ e ao conhecimento dos indivíduos sobre TEV e tromboprofilaxia $(14,3 \%)^{(16)}$.

A avaliação de risco de TEV pelo enfermeiro ainda não é rotina na maioria dos serviços ${ }^{(16,28)}$. Contudo, com a elaboração de protocolos institucionais e treinamentos, a enfermagem deve apropriar essas ferramentas e incluí-las em sua rotina ${ }^{(16,28)}$.

A Classificação Internacional das Intervenções de Enfermagem apresenta um conjunto de intervenções Precaução contra embolia, aplicáveis ao diagnóstico de enfermagem Perfusão Tissular Periférica Ineficaz (e o correspondente de Risco ${ }^{(30)}$. Neste conjunto de Intervenções de Enfermagem, as atividades são divididas entre cuidados com pacientes em profilaxia farmacológica e mecânica, sendo apresentadas a seguir as que têm relação direta com a profilaxia mecânica ${ }^{(30)}$ :

- Iniciar o regime apropriado de TEV em pacientes de risco imediatamente, de acordo com a política e protocolo organizacional;

- Elevar qualquer membro que se suponha estar afetado $20^{\circ}$ ou mais acima do nível do coração para, assim, aumentar o retorno venoso;

- Aplicar MECG para reduzir o risco de TVP ou para evitar sua recorrência, de acordo com a política e o protocolo organizacional;

- Manter MECG para evitar o desenvolvimento de síndrome pós-trombótica, que é causada por coágulos de longa data na extremidade afetada e fluxo venoso reduzido;

- Aplicar dispositivo de CPI, de acordo com a política e com o protocolo organizacional;

- Remover MECG e dispositivo de compressão pneumática intermitente por 15 a 20 minutos, a cada 8 horas ou de acordo com a política, e com o protocolo organizacional;
- Auxiliar o paciente com a amplitude de movimentação passiva ou ativa, conforme apropriado;

- Encorajar o paciente a flexionar e estender o pé e as pernas pelo menos 10 vezes a cada hora;

- Mudar a posição do paciente a cada 2 horas, encorajar a movimentação ou a deambulação precoce, de acordo com o que o paciente tolerar;

- Evitar massagear ou comprimir os músculos dos membros afetados;

- Orientar o paciente que ele não pode cruzar as pernas e que deve evitar ficar sentado por longos períodos com as pernas pendentes.

As Intervenções de Enfermagem são submetidas a um processo de validação para comporem o catálogo da NIC. Contudo, os guidelines apenas indicam ou contraindicam as intervenções e, nesse caso, não descrevem a utilização das meias ou de dispositivos de CPI, à exceção de um guideline mais antigo do The National Institute of Health and Clinical Excellence (NICE) ${ }^{(8)}$ e de um documento do Instituto Joanna Briggs, publicado em $2018^{(31)}$. Trata-se de um documento que orienta o uso de meias compressivas, com um sumário de evidências acerca do tema a ser consultado por enfermeiros, para implementação de melhores práticas baseadas em evidência ${ }^{(31)}$. Com base nestas duas últimas referências, cabe ainda citar, em relação ao uso de meias e dispositivos de $\mathrm{CPI}^{(8,31)}$ :

- As meias de compressão não devem ser oferecidas a pacientes: com suspeita de doença arterial periférica, by-pass arterial periférico, neuropatia periférica ou outra causa de prejuízo sensorial, dermatites ou lesões ou fragilidades na pele dos MMII, alergia ao material, insuficiência cardíaca congestiva e deformações nos MMII;

- Em formação de edema, o membro deve ser medido novamente e a meia ajustada;

- Incentivar os pacientes para o uso de meias dia e noite, até que eles não tenham mais a mobilidade significativamente reduzida;

- Remover diariamente as meias para fins de higiene e inspecionar a condição da pele;

- Interrompa o uso de meias, se houver marcação, formação de bolhas ou descoloração da pele, particularmente sobre os calcanhares e proeminências ósseas, ou se o paciente sente dor ou desconforto;

- Mostrar aos pacientes como usar corretamente as meias e garantir que eles entendam que isso reduzirá seu risco de desenvolvimento de TEV;

- Monitorar o uso de meias compressivas e oferecer assistência se elas não estiverem sendo usadas corretamente.

Os pacientes devem ser avaliados pela enfermagem como rotina e norteada pelos protocolos institucionais, além do mais, as medidas de prevenção devem ser implementadas. Quanto às medidas farmacológicas, cabe ao enfermeiro a dupla checagem. As medidas não farmacológicas são de competência do enfermeiro, devendo ser 
implementadas com iniciativa, pautada em evidências, respaldadas por protocolos e sem aguardar a prescrição de outro profissional. No centro cirúrgico, por exemplo, na admissão pré-operatória imediata o enfermeiro deve avaliar o risco para TEV e assegurar as medidas intraoperatórias de prevenção.

O guideline da AORN orienta que os enfermeiros do centro cirúrgico estão em posição de garantir que os pacientes sejam avaliados para o risco de TEV, que devem iniciar as medidas preventivas na admissão do paciente, participar da discussão das necessidades e da seleção de medidas de profilaxia adequadas em cada caso, colaborando com outros membros da equipe cirúrgica e garantindo a indicação correta do uso das medidas não farmacológicas ${ }^{(31)}$.

\section{CONSIDERAÇÕES FINAIS}

O tromboembolismo venoso é um evento passível de atenção e cuidados para a prevenção. Por isso, a avaliação para estratificação de risco deve ser realizada para todos os pacientes cirúrgicos e a profilaxia mecânica deve ser instituída para pacientes em condições específicas, associada à profilaxia farmacológica.

Por fim, os enfermeiros cirúrgicos devem incluir em suas atividades as avaliações de risco e instituírem as medidas não farmacológicas, baseadas em evidências científicas e protocolos institucionais bem delineados. Sendo assim, o uso de meias elásticas de compressão graduada e compressão pneumática intermitente, com indicações baseadas em evidências, deve ser assegurado pelos enfermeiros.

\section{RESUMO}

Objetivo: O estudo teórico apresentado tem por objetivo discorrer sobre o papel do enfermeiro na prevenção mecânica do tromboembolismo venoso em pacientes cirúrgicos. Método: $\mathrm{O}$ estudo considerou as versões atualizadas dos principais guidelines internacionais e revisões acerca do tema. Além de serem discutidas as medidas não farmacológicas de prevenção de tromboembolismo venoso em pacientes cirúrgicos e o papel do enfermeiro. Resultados: É importante que o enfermeiro cirúrgico inclua, em suas atividades, as avaliações de risco do tromboembolismo venoso e as medidas profiláticas não farmacológicas, baseadas em evidências científicas e protocolos institucionais bem delineados. Conclusão: Dentre as medidas mecânicas, o uso de meias elásticas de compressão graduada e a compressão pneumática intermitente devem ser asseguradas pelos enfermeiros.

\section{DESCRITORES}

Tromboembolia; Tromboembolia Venosa; Segurança do Paciente; Procedimentos Cirúrgicos Operatórios; Enfermagem de Centro Cirúrgico.

\section{RESUMEN}

Objetivo: El estudio teórico presentado tiene como objetivo discurrir sobre el papel del enfermero en la prevención mecánica del tromboembolismo venoso de pacientes quirúrgicos. Método: El estudio tuvo en cuenta las versiones actualizadas de las principales directrices y revisiones internacionales sobre el tema, además de discutir las medidas no farmacológicas para la prevención del tromboembolismo venoso en pacientes quirúrgicos y el papel del enfermero. Resultados: Es importante que el enfermero quirúrgico incluya, en sus actividades, evaluaciones de riesgo de tromboembolismo venoso y medidas profilácticas no farmacológicas, basadas en la evidencia científica y en protocolos institucionales bien diseñados. Conclusión: Entre las medidas mecánicas, los enfermeros deben garantizar el uso de medias elásticas de compresión graduada y la compresión neumática intermitente.

\section{DESCRIPTORES}

Tromboembolia; Tromboembolia Venosa; Seguridad del Paciente; Procedimientos Quirúrgicos Operativos; Enfermería de Quirófano.

\section{REFERÊNCIAS}

1. Grosse SD, Nelson RE, Nyarko KA, Richardson LC, Raskob GE. The economic burden of incident venous thromboembolism in the United States: A review of estimated attributable healthcare costs. Thromb Res. 2016;137:3-10. https://doi.org/10.1016/j.thromres.2015.11.033

2. Qaseem A, Chou R, Humphrey LL, Starkey M, Shekelle P; Clinical Guidelines Committee of the American College of Physicians. Venous thromboembolism prophylaxis in hospitalized patients: a clinical practice guideline from the American College of Physicians. Ann Intern Med. 2011;155(9):625-32. https://doi.org/10.7326/0003-4819-155-9-201111010-00011

3. Raymundo SR, Lobo SM, Hussain KM, Hussein KG, Secches IT. What has changed in venous thromboembolism prophylaxis for hospitalized patients over recent decades: review article. J Vasc Bras. 2019;18:e20180021. https://doi.org/10.1590/1677-5449.002118

4. Afshari A, Fenger-Eriksen C, Monreal M, Verhamme P; ESA VTE Guidelines Task Force. European guidelines on perioperative venous thromboembolism prophylaxis: mechanical prophylaxis. Eur J Anaesthesiol. 2018;35(2):112-5. https://doi.org/10.1097/ EJA.0000000000000726

5. Samama CM, Afshari A; ESA VTE Guidelines Task Force. European guidelines on perioperative venous thromboembolism prophylaxis. Eur J Anaesthesiol. 2018;35(2):73-6. https://doi.org/10.1097/EJA.0000000000000702

6. Farhat FC, Gregório HC, Carvalho RD. Evaluation of deep vein thrombosis prophylaxis in a general hospital. JVasc Bras. $2018 ; 17(3)$ :184-92. [https://doi.org/10.1590/1677-5449.007017

7. Gould MK, Garcia DA, Wren SM, Karanicolas PJ, Arcelus JI, Heit JA, et al. Prevention of VTE in nonorthopedic surgical patients: Antithrombotic Therapy and Prevention of Thrombosis, 9th ed: American College of Chest Physicians Evidence-Based Clinical Practice Guidelines. Chest. 2012;141(2 Suppl):e227S-77S. https://doi.org/10.1378/chest.11-2297

8. Hill J, Treasure T; Guideline Development Group. Reducing the risk of venous thromboembolism (deep vein thrombosis and pulmonary embolism) in patients admitted to hospital: summary of the NICE guideline. Heart. 2010;96(11):879-82. https://doi.org/10.1136/hrt.2010.198275

9. Link T. Guideline implementation: prevention of venous thromboembolism: 1.6 www.aornjournal.org/content/cme. AORN J. 2018;107(6):737-48. https://doi.org/10.1002/aorn.12146 
10. Sachdeva A, Dalton M, Amaragiri SV, Lees T. Graduated compression stockings for prevention of deep vein thrombosis. Cochrane Database Syst Rev. 2014;(12):CD001484 https://doi.org/10.1002/14651858.CD001484.pub3

11. Pavon JM, Adam SS, Razouki ZA, McDuffie JR, Lachiewicz PF, Kosinski AS, et al. Effectiveness of intermittent pneumatic compression devices for venous thromboembolism prophylaxis in high-risk surgical patients: a systematic review. J Arthroplasty. 2016;31(2):524-32. https://doi.org/10.1016/j.arth.2015.09.043

12. Ayhan $H$, lyigun E, Ince S, Can MF, Hatipoglu S, Saglam M. A randomised clinical trial comparing the patient comfort and efficacy of three different graduated compression stockings in the prevention of postoperative deep vein thrombosis. J Clin Nurs. 2015;24(15-16):2247-57. https://doi.org/10.1111/jocn.12866

13. National Institute for Health and Clinical Excellence. Venous thromboembolism: reducing the risk of venous thromboembolism (deep vein thrombosis and pulmonary embolism) in patients admitted to hospital. London: Royal College of Physicians; 2010 [cited 22 May 2020 ]. Available from: https://www.ncbi.nlm.nih.gov/pubmed/23346611

14. Sajid MS, Desai M, Morris RW, Hamilton G. Knee length versus thigh length graduated compression stockings for prevention of deep vein thrombosis in postoperative surgical patients. Cochrane Database Syst Rev. 2012;5(5):CD007162. https://doi.org/10.1002/14651858. CD007162.pub2

15. Vitor SK, Daou JP, Góis AF. Prevenção de tromboembolismo (trombose venosa profunda e embolia pulmonar) em pacientes clínicos e cirúrgicos. Diagn tratamento [Internet]. 2016 [cited 22 May 2020];21(2):59-64. Available from: http://files.bvs.br/upload/S/1413-9979/2016/ v21n2/a5583.pdf

16. Barp M, Carneiro VSM, Amaral KVA, Pagotto V, Malaquias SG. Nursing care in the prevention of venous thromboembolism: an integrative review. Rev Eletr Enf. 2018;20:v20a14. https://doi.org/10.5216/ree.v20.48735

17. Urbankova J, Quiroz R, Kucher N, Goldhaber SZ. Intermittent pneumatic compression and deep vein thrombosis prevention: a metaanalysis in postoperative patients. Thromb Haemost. 2005;94(6):1181-5. https://doi.org/10.1160/TH05-04-0222

18. Zareba P, Wu C, Agzarian J, Rodriguez D, Kearon C. Meta-analysis of randomized trials comparing combined compression and anticoagulation with either modality alone for prevention of venous thromboembolism after surgery. Br J Surg. 2014;101(9):1053-62. https://doi.org/10.1002/bjs.9527 PMID:24916118

19. Morris RJ, Woodcock JP. Intermittent pneumatic compression or graduated compression stockings for deep vein thrombosis prophylaxis? A systematic review of direct clinical comparisons. Ann Surg. 2010;251(3):393-6. https://doi.org/10.1097/SLA.0b013e3181b5d61c

20. Arabi YM, Khedr M, Dara SI, Dhar GS, Bhat SA, Tamim HM, et al. Use of intermittent pneumatic compression and not graduated compression stockings is associated with lower incident VTE in critically ill patients: a multiple propensity scores adjusted analysis. Chest. 2013;144(1):152-9. https://doi.org/10.1378/chest.12-2028

21. Kakkos SK, Caprini JA, Geroulakos G, Nicolaides AN, Stansby G, Reddy DJ, et al. Combined intermittent pneumatic leg compression and pharmacological prophylaxis for prevention of venous thromboembolism. Cochrane Database Syst Rev. 2016;9(9):CD005258. https://doi. org/10.1002/14651858.CD005258.pub3

22. Cohen AT, Tapson VF, Bergmann JF, Goldhaber SZ, Kakkar AK, Deslandes B, et al.; ENDORSE Investigators. Venous thromboembolism risk and prophylaxis in the acute hospital care setting (ENDORSE study): a multinational cross-sectional study. Lancet. 2008;371(9610):387-94https:// doi.org/10.1016/S0140-6736(08)60202-0

23. Lopes BA, Teixeira IP, Souza TD, Tafarel JR. Do we know how to prescribe venous thromboembolism prophylaxis to hospitalized patients?. J Vasc Bras. 2017;16(3):199-204. https://doi.org/10.1590/1677-5449.008516

24. Caprini JA. Risk assessment as a guide for the prevention of the many faces of venous thromboembolism. Am J Surg. 2010;199(1, Supl):S3-10. https://doi.org/10.1016/j.amjsurg.2009.10.006

25. Maffei FH, Caiafa JS, Ramacciotti E, Castro AA. Normas de orientação clínica para prevenção, diagnóstico e tratamento da trombose venosa profunda. J Vasc Bras [Internet]. 2005 [cited 2020 May 22];4(Supl 3):S205-20. Available from: http://www.saudedireta.com.br/ docsupload/1334451524Arquivo_4.pdf

26. Streiff MB, Carolan HT, Hobson DB, Kraus PS, Holzmueller CG, Demski R, et al. Lessons from the Johns Hopkins Multi-Disciplinary Venous Thromboembolism (VTE) Prevention Collaborative. BMJ. 2012;344 jun19 6:e3935. https://doi.org/10.1136/bmj.e3935

27. Kahn SR, Morrison DR, Diendéré G, Piché A, Filion KB, Klil-Drori AJ, et al. Interventions for implementation of thromboprophylaxis in hospitalized patients at risk for venous thromboembolism. Cochrane Database Syst Rev. 2018;4:CD008201. [ https://doi. org/10.1002/14651858.CD008201.pub3

28. Brewer CF, Ip D, Drasar E, Aghakhani P. Reducing inappropriately suspended VTE prophylaxis through a multidisciplinary shared learning programme and electronic prompting. BMJ Open Qual. 2019;8(1):e000474. https://doi.org/10.1136/bmjoq-2018-000474

29. Duff J, Walker K, Omari A. Translating venous thromboembolism (VTE) prevention evidence into practice: a multidisciplinary evidence implementation project. Worldviews Evid Based Nurs. 2011;8(1):30-9. [https://doi.org/10.1111/j.1741-6787.2010.00209.x

30. McCloskey JC, Bulecheck GM. Classificação das Intervenções de enfermagem. 6 ed. Porto Alegre: Artmed; 2016.

31. Long Khanh-Dao Le. Evidence summary. Graduated compression stockings: clinician information. The Joanna Briggs Institute EBP Database, JBI@Ovid. 2018; JBI179. 